\title{
Wide-Area Network State Monitoring System Based On LoRa Communication
}

\author{
Abdelmadjid RECIOUI, Yasser MOKHBI and Aymen MECHERI \\ Laboratory of Signals and Systems, Institute of Electrical and electronic Engineering, University \\ M'hamed Bougara of Boumerdes, Avenue de l'indépandance, 35000, Boumerdes, Algeria
}

\begin{abstract}
To meet the requirements of long range, small amount of data transmission, better data correctness and less delay, a wide-Area Network State Monitoring System approach based on LoRa communication is discussed in our Project. The objective is to study and test the performance of Two communication techniques used in wide area monitoring system, a single input single output (SISO) system and a multiple input multiple output system (MIMO) in terms ofBit Error Rate(BER) with varying the signal to noise Ratio(SNR).In addition, we study the Wide Area Monitoring system based on LoRa communication. The IEEE 14-bus power system with Phasor Measurement Units(PMUs) placed at selected buses is considered. The simulation results demonstrate that the MIMO system overtake the SISO system for better Bit Error Rate. Furthermore, the value of the reporting rate has been varied for both systems in MATLAB to see the effect on the data correctness and the results show that the Bit Error Rate value decreases with the increase of reporting rate. Finally, we dealt with a modified LoRa MATLAB code emulator to investigate the data error and delay. The results show that LoRa Technology has better performance in terms of delay and error.
\end{abstract}

Keywords : WAMS, State Monitoring, Communication, SISO, MIMO, LoRa, BER

\section{Introduction}

Power system is becoming more and more complex, mainly due to the increase of new technologies and the demand of using clean energy. Wide-area monitoring, wide-area control and wide-area protection utilize Wide Area Networks turn out to be the nextgeneration key to improve power system planning. The modern power grid is considered to be the most complex human-made system, which is monitored by wide-area monitoring system. Providing time-synchronized data of power system operating states, wide-area monitoring system will play a crucial role in next generation smart grid protection and control. wide-area monitoring system helps secure efficient energy transmission as well as reliable and optimal grid management. As the key enabler of a smart grid, numerous sensors such as PMU and current sensors transmit real-time dynamic data, which is usually protected by encryption algorithm from malicious attacks, over wide-area-network to power system control centers so that monitoring and control of the whole system is possible [1-3].

Nowadays, high data rate techniques have gained considerable interests in communication systems. Electric grid was designed to operate as a vertical structure consisting of generation, transmission, and distribution and supported with controls and devices to maintain reliability, stability, and efficiency. However, system operators are now facing new challenges including the penetration of Renewable Energy Resource in the legacy system, rapid technological change, and different types of market players and end users. The next iteration, the smart grid, will be equipped with communication support schemes and real-time measurement techniques to enhance resiliency and forecasting as well as to protect against internal and external threats. The design framework of the smart grid is based upon unbundling and restructuring the power sector and optimizing its assets [4-5].

With the rapid growth of IOT technology, it has been applied to an increasing number of fields, such as intelligent agriculture, industrial automation, smart city, smart home, etc. [6]. Currently, the most widely used ZigBee, Bluetooth, 2G, 3G, 4G, and other communication technologies have their own pros and cons [7][8]. Xing et al. proposed a greenhouse intelligent information monitoring system using ZigBee wireless sensor. This system used ZigBee wireless sensor to collect field environmental parameters and realized centralized monitoring, data display, data storage, and data mining [9]. Shen et al. enabled traffic information detection using Bluetooth communication, i.e., to transmission of vehicle position, speed, etc. information via Bluetooth [10]. Zhang et al. proposed wireless sensor monitoring nodes powered by solar panels and realized real-time, accurate, and remote transmission data using GPRS technology [11]. However, ZigBee and Bluetooth are short-range radio technologies and are not suitable for long-range transmission scenarios. 2G, 3G, 4G, and other solutions based on cellular communication can 
provide a wider coverage, but they consume too much energy and increase the operating costs [8].

To solve these problems, LPWAN has emerged and gained wide attention from researchers which have analyzed low-power and long-range wireless technology, implemented a networking scheme and tested the performance of LoRa wireless transmission technology [12]. Shi et al. proposed a smart parking system using NB-loT communication technology, which can effectively improve the utilization rate of the existing parking facilities [13]. Anand et al. presented a remote monitoring mechanism for the water level in a storage tank using NB-loT [14]. The above studies show that LoRa has the advantages of high stability, ultralong transmission, ultralow power, and low cost [15].

Wide-area monitoring, wide-area control and wide-area protection utilize Wide Area Networks and turn out to be the nextgeneration key to improve power system planning, operation and protection in the smart grid. These applications employ the system broad data and wisely chosen local information to oppose the spread of harming disturbances [16]. Wide-area monitoring, control and protection applications present higher data resolution and shorter response time than the classical supervisory control and data acquisition (SCADA) and energy management (EMS) systems. Wide-area monitoring, control and protection applications provide highresolution data contrary to SCADA/EMS which offers a measurement update interval of several seconds (or even minutes) [17].

$$
\text { The IEEE Standard for }
$$

Synchrophasors for Power Systems (IEEE Std.C37.118 provides definitions of measurement and data transmission formats for real-time data reporting in electric power systems [18]. For wide-area monitoring applications, the size containing measurements made by a PMU has a minimum message of 52 bytes. The required response time for wide area monitoring applications is in the range of milliseconds to minutes, and the requirement on communication system reliability is very high. Due to the importance of the communication infrastructure in the success of smart grid implementation and operation, there has been a variety of research works in the literature focusing on this topic, so the suitable communication technologies for transmissionlevel systems for a long- distance are important [19].
Multiple-input multiple output (MIMO) techniques are being widely adopted in the current fourth generation (4G) telecommunication systems and they are expected to be a key technologies for the fifthgeneration (5G) communications. MIMO systems take advantage of the multipath nature of the propagation channel. However, the antenna properties turn out to affect correlations

among channel coefficients. MIMO, as a currently well established technology, offers considerable benefits, such as improving link quality and largely attainable data rates [2021]. When antenna array elements are made closer one another, the effect of electromagnetic mutual coupling between them becomes a common phenomenon. The mutual coupling can dangerously deteriorate the performance of the array in the form of signalto-interference-noise ratio (SINR) reduction and the signal processing algorithm nonconvergence [22-23]. It precisely degrades some parameters such as the carrier frequency offset [24], channel [25], and angle of arrival estimations [26]. Also, the awful effect of mutual coupling on the active reflection coefficient of a MIMO antenna is another result that cannot be ignored [27]. Furthermore, the active voltage standing wave ratio (VSWR) may reach intolerable values. Despite the negative effects of mutual coupling. the MIMO system performance deterioration, it can be exploited for array calibrations as in [28-29]. The mutual coupling modifies the antenna characteristics in an array, and hence affects the MIMO system performance (e.g., capacity, error rate, and spectral re-growth). The system performance can be partly improved by adjusting out the mutual coupling using digital techniquesbut without improving the SINR. Thus, it is very imperative to lessen the mutual coupling when it comes to MIMO antenna design.

The aim of this paper is to use different communication technologies to improve data transfer in terms of data completeness and correctness in Wide Area Monitoring systemby using the software MATLAB for the simulation.We started by two spatial diversity techniques which are SISO and MIMO systems after that we mainly studied the transmitted and the received signal of the IEEE-14 bus standard power system based on LoRa emulator code on MATLAB.

\section{WAMS COMMUNICATION SYSTEMS}

\subsection{SISO and MIMO systems}

Modern wireless communication demands constant improved spectral efficiency. More 
number of users is needed to be accommodated in a given bandwidth with high quality standards for communication. Different diversity techniques are used for it. Spatial diversity deals with multiple number of transmitting and receiving antenna at transmitter and receiver respectively. When the same signal is transmitted or received via multiple devices, spatial diversity is formed.

SISO is the simplest antenna technology in which one antenna is used at the source (transmitter) and one antenna is used at the destination (receiver). As no diversity is applicable in SISO case, multipath fading[29] is a prominent issue in this category of communication [30-32].

The most significant technological developments in communication are using Multiple Input Multiple Output (MIMO) antenna architectures [33]. In a MIMO system, multiple element antenna arrays are deployed at both the transmitter and the receiver. The suitability of a MIMO wireless system for spatial multiplexing is largely dependent on the characteristics of the wireless channel, the antenna configuration and the ability of the receiver to accurately recover the channel coupling matrix coefficients. The communications challenge lies in designing the sets of signals simultaneously sent by the transmit antennas and the algorithms for processing those observed by the receive antennas, so that the quality of the transmission (i.e., bit error probability) and/or its data rate are superior to those supported by traditional single antenna systems [34]. These gains can then provide increased reliability, reduced power requirements and higher composite data rates. What is especially exciting about the benefits offered by MIMO technology is that they can be attained without the need for additional spectral resources, which are not only expensive but also extremely scarce.

MIMO has been shown to offer significant improvements in spectral efficiency and link reliability through spatial multiplexing and space-time coding, respectively. Under conducive

channel conditions (such as rich scattering), the receiver exploits differences in the spatial signatures of the multiplexed streams to separate the different signals, thereby realizing a capacity gain.

\subsection{Performance Measures}

Signal -to-noise ratio (SNR) is defined as the ratio of the desired signal power to noise power. SNR indicates the reliability of link between the transmitter and receiver. In OFDM based multicarrier modulation system, the channel bandwidth is wide and interference is not constant over the whole band in use. It is very likely that there is variation of spectral content over the OFDM sub carriers i.e. some part of spectrum is more affected by interference than the other parts of spectrum The most meaningful criterion for evaluation of performance of communication systems is the bit error rate(BER). The Bit Error Rate (BER) is the number of acceptable errors you are prepared to tolerate. This ratio is closely linked to the Signal-to-Noise-Ratio (SNR) which is measured in decibels (dB).In case of orthogonal communications systems, the main sources affecting its BER performance are additive white Gaussian noise(AWGN) and Inter-carrier Interference $(\mathrm{ICl})$. In most of the cases, the $\mathrm{ICl}$ is assumed to have a normal distribution; however such assumption is not accurate theoretically. With the help of well developed conventional single carrier communication systems, the BER expression of OFDM systems can be obtained without knowing the $\mathrm{ICl}$ distribution function.

\subsection{Description of LoRa Technology:}

As one of loT communication technologies, LoRa is a kind of ultra-long distance wireless transmission technology based on the spread spectrum transmission techniques and CSS (chirp spread spectrum) modulation, which is one of the loT communication technologies. Its name comes from the abbreviation of "long range". It can be seen from the name, the biggest feature of LoRa is the long distance communication. LoRa's spread spectrum technology changes the balance between transmission power consumption and transmission distance, and completely changes the situation in the field of embedded wireless communication. This technology presents a new communication technology that enables long-distance, long battery life, large system capacity and low hardware costs to meet the loT needs [35].

Table 1 compares some parameters including transfer rate, transmission range, power consumption and cost between some popular wireless technologies. Accordingly, LoRa has shown its superiority in many aspects. Its only weakness is the data rate. However, in wireless sensor network applications, this is not an issue. 
Table 1 Comparison of some wireless technology [36]

\begin{tabular}{|c|c|c|c|c|c|}
\hline & $\begin{array}{c}\mathrm{Tx} \\
\text { Range }\end{array}$ & $\begin{array}{c}\text { Tx } \\
\text { Rate }\end{array}$ & $\begin{array}{c}\text { Tx } \\
\text { Power }\end{array}$ & $\begin{array}{l}\text { Sleep } \\
\text { Power }\end{array}$ & cost \\
\hline Bluetooth & $15 \mathrm{M}$ & $\begin{array}{c}3 \\
\text { Mbps }\end{array}$ & $\begin{array}{l}20 \\
\mathrm{~mA}\end{array}$ & $16 \mu A$ & low \\
\hline $\begin{array}{c}\text { Wifi } \\
\text { (CC3200) }\end{array}$ & $\begin{array}{c}150 \\
M\end{array}$ & $\begin{array}{c}3 \\
\mathrm{Mbp}\end{array}$ & $\begin{array}{l}75 \\
\mathrm{~mA}\end{array}$ & $\begin{array}{l}3.5 \\
\mu A\end{array}$ & high \\
\hline $\begin{array}{l}3 \mathrm{G} / 4 \mathrm{G} \\
\text { (U8300) }\end{array}$ & & $\begin{array}{c}14 \\
\mathrm{Mbp}\end{array}$ & $\begin{array}{l}800 \\
\mathrm{~mA}\end{array}$ & $50 \mu A$ & high \\
\hline $\begin{array}{l}\text { ZigBee } \\
\text { (REX } \\
\text { 3DP) }\end{array}$ & $\begin{array}{c}100- \\
200 \\
M\end{array}$ & $\begin{array}{c}250 \\
\text { Kbps }\end{array}$ & $\begin{array}{l}200 \\
\mathrm{~mA}\end{array}$ & $\begin{array}{l}0.4 \\
\mu A\end{array}$ & low \\
\hline $\begin{array}{c}\text { LoRa } \\
\text { (SX1278) }\end{array}$ & $\begin{array}{c}3000 \\
M\end{array}$ & $\begin{array}{c}2.4 \\
\text { Kbps }\end{array}$ & $\begin{array}{l}110 \\
\mathrm{~mA}\end{array}$ & $\begin{array}{l}2.0 \\
\mu A\end{array}$ & Iow \\
\hline
\end{tabular}

LoRa was developed by Semtech to work in 433.868 or $915 \mathrm{MHz}$ ISM Band (depend on the regional placement) with transmission rate between $0.25 \mathrm{kbps}-50 \mathrm{kbps}$ [37]. The parameters that possibly changed in LoRa modulation are Bandwidth, Spreading Factor and Code Rate. The spreading factors are - in short - the duration of the chirp. LoRa operates with spread factors from 7 to 12 . SF7 is the shortest time on air and SF12 will be the longest. Each step up in spreading factor doubles the time on air to transmit the same amount of data [38] [39].

\section{SIMULATION RESULTS AND DISCUSSIONS}

\subsection{System description}

The work considers a small scale wide area monitoring system, the power system used in our work is the IEEE 14 bus standard system, the placement of PMUs is important as we want to make the entire system observable, with zero injection at bus 7 , only 3 PMUs placed at bus 2,6 and 9 are needed.

SISO and MIMO communication Simulink models are considered. The data collected from each PMU is sent through both SISO and MIMO systems. The three PMUs are synchronized using GPS clock. The state of all the power system is estimated using the received data from these three PMUs, to compare the performance of both SISO and MIMO Systems in terms of Bit Error Rate ; BER (data correctness). The SNR (signal to noise ratio) with an additive White Gaussian Noise (AWGN) block was varied and the effect of this change on the BER is investigated.

The measurement are sent by using the Function LoRa_Tx and received by the function LoRa_Rx on MATLAB which are an open source code in [40]. The work is based on a modified pilot file that is changed into 3 different files to allow transferring of real values measurement's for the $2^{\text {nd }}$ bus, $6^{\text {th }}$ bus and the $9^{\text {th }}$ bus.

\subsection{Results and Discussions}

Performing simulations based on the Simulink model and the Pilot files produces figures 1, 2 and 3 for buses 2, 6 and 9 in terms of amplitude, phase and frequency, respectively.

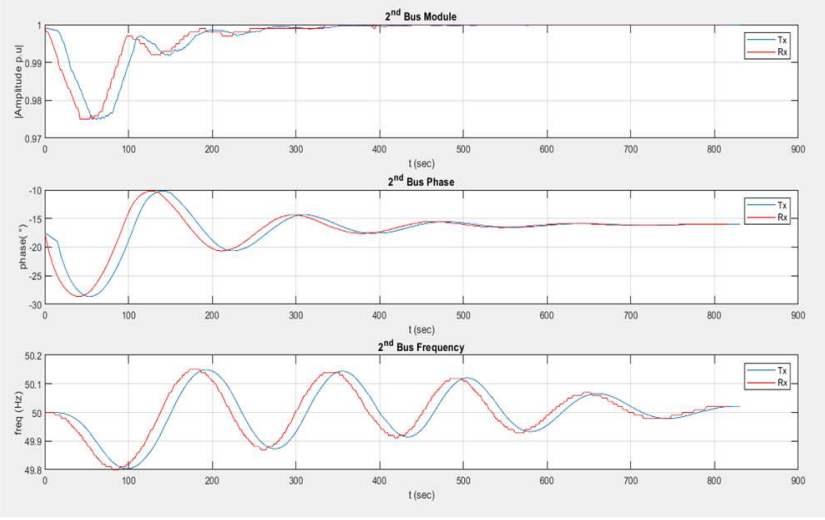

Fig. 1 Amplitude, Phase and Frequency for $2^{\text {nd }}$ bus.

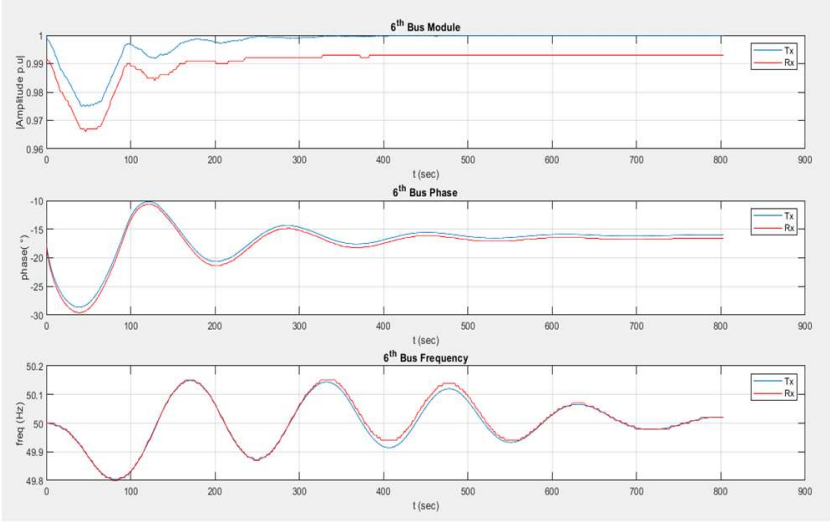

Fig. 2 Amplitude, Phase and Frequency for $6^{\text {th }}$ bus.

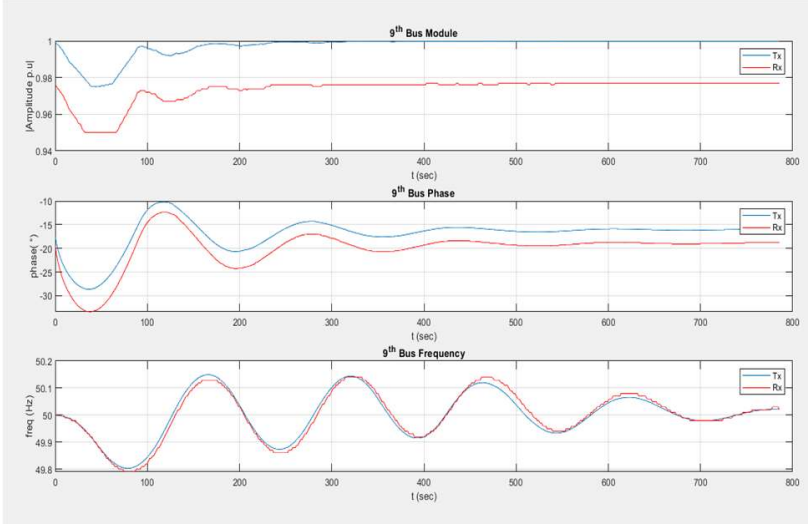

Fig. 3 Amplitude, Phase and Frequency for $9^{\text {th }}$ bus.

From the above figures, we can see that the transmitted signal is approximately the same as the received one, with a slight change at some different period of time. The simulation time is 42.000000 min and $14.400000 \mathrm{sec}$ with 
a decision instant of $1.483674 \mathrm{sec}$. Table 2 gives the time delay for each bus and their corresponding transmission error.

Table 2 The error and delay for each bus

\begin{tabular}{|l|c|c|}
\cline { 2 - 3 } \multicolumn{1}{c|}{} & Time delay (sec) & Error \\
\hline $2^{\text {nd }}$ bus & 0.845881 & 0.049267413 \\
\hline $6^{\text {th }}$ bus & 0.805521 & 0.046134732 \\
\hline $9^{\text {th }}$ bus & 0.786660 & 0.037471326 \\
\hline
\end{tabular}

The data in the above table shows that the time delay and the error for each bus is achieving good results for the same reporting rate at different buses in the system.

\section{The effect of varying the reporting rate on error and delay:}

We have changed the reporting rate by changing the reporting rate factor $k=4$. The following results are obtained. The simulation time is $39.000000 \mathrm{~min}$ and $0.631000 \mathrm{sec}$ with a decision instant of $1.459555 \mathrm{sec}$.

Table 3 The error and delay for each bus for a reporting rate factor $k=4$

\begin{tabular}{|c|c|c|}
\multicolumn{1}{c|}{} & \multicolumn{1}{c|}{$\begin{array}{c}\text { Teporting rate factor } k=4 . \\
(\mathrm{sec})\end{array}$} & Error \\
\cline { 2 - 3 } \multicolumn{1}{c|}{} & 0.783991 & 0.016123796 \\
\hline $2^{\text {nd }}$ bus & 0.768762 & 0.015199478 \\
\hline $6^{\text {th }}$ bus & 0.767647969 \\
\hline $9^{\text {th }}$ bus & 0.774819 & 0.016477569 \\
\hline
\end{tabular}

From Table 3, we can see that the time delay and error decrease as we change the reporting rate factor which lead to a new value of the reporting rate. The increase of the reporting rate affect the time delay and error positively which we can say that the interval length at which the event will be reported should been taken into consideration for achieving better time delay and data error.

It should be noted that for the same Signal to Noise Ratio, it can be clearly seen that the MIMO system outperforms the SISO System in terms of data correctness (BER) which recommends its deployment in WAMS. As an example, The BER for the $2^{\text {nd }}$ bus increases from 0.348893984308837 for $S N R=0$ to 0.441029359724782 for $S N R=30$ in the SISO system. However, it rises from 0.137060844986704 for $S N R=0$ to 0.208282807752671 for $S N R=30$ in the case of the MIMO system.

\section{CONCLUSION}

In this paper, communication techniques used in wide area monitoring systems have been investigated with focus on LoRa communication technology. The performance of the LoRa communication in terms of Bit Error Rate has been studied for both Single Input Single Output (SISO) and Multiple Input and Multiple Output (MIMO) systems. The transmitted and received signal based on LoRa modulation and coding scheme are almost identical which demonstrates the benefit of the LoRa communication technology over the other technologies for achieving better error and delay time. The simulation results demonstrate that the MIMO system is an option to adopt compared to the SISO system as better BER is achieved for the same SNR. Also, if blended with the LoRa technology, the results show that this Technology is more practical which is benefitial to the design of better communication infrastructure in wide area systems.

\section{References}

[1] Akash K Singh "Trust and Trust Management Models for Ecommerce \& Sensor Network" International Journal of Computational Engineering Research (ijceronline.com) Vol. 2 Issue. 7. pp-585-619.

[2] Abdelmadjid Recioui and Hamid Bentarzi, Optimizing smart grid operation and control, IGI Global, 2021.

[3] A. Recioui, "Application of Teaching LearningBased Optimization to the Optimal Placement of Phasor Measurement Units", Book chapter in: Handbook of Research on Emergent Applications of Optimization Algorithms, IGI Global, 2018.

[4] Afaf Saoud and Abdelmadjid Recioui, "A review on Data communication in smart grids Algerian Journal of Signals and Systems, Vol.2, Issue 3, September 2017. pp:162-179.

[5] J. Momoh, "SMART GRID Fundamentals of Design and Analysis," IEEE Book and Information Services (BIS).2012.

[6] F. D. V. Abeele, J. Haxhibeqiri, I. Moerman and J. Hoebeke, "Scalability analysis of largescale LoRaWAN networks in ns-3," IEEE Internet of Things Journal, vol. 4, no. 6, pp. 2186 - 2198, Dec. 2017.

[7] A. Tzounis, N. Katsoulas, T. Bartzanas and C. Kittas, "Internet of Things in agriculture, recent advances and future challenges," Biosystems Engineering, vol. 164, no. 2017, pp. 31 - 48, Dec. 2017

[8] K. Mekki, E. Bajic, F. Chaxel and Fernand Meyer, "A comparative study of LPWAN technologies for large-scale loT deployment," ICT Express. https://doi.org/10.1016/j.icte.2017.12.005.

[9] X. J. Xing, J. C. Song, L. Y. Lin, M. Q. Tian and Z. P. Lei, "Development of intelligent information monitoring system in greenhouse based on wireless sensor network," in International Conference on Information Science and Control Engineering. IEEE Computer Society, 2017, pp. 970- 974.

[10] X. P. Shen, X. Wang and M. Jia, "Design and implementation of traffic information detection equipment based on Bluetooth communication," in IEEE, Information Technology, Networking, Electronic and Automation Control Conference, 2017, pp. $1595-1601$.

[11] X. H. Zhang, J. L. Du, C. G. Fan, D. Liu, J. L. Fang and L. S. Wang, "A wireless sensor monitoring node based on automatic tracking solar-powered panel for paddy field environment," IEEE Internet of Things 
Journal, vol. 4, no. 5, pp. 1304 - 1311, Oct. 2017.

[12] M. S. A. Muthanna, M. M. A. Muthanna, A. Khakimov and A. Muthanna, "Development of intelligent street lighting services model based on LoRa technology," in ElConRus, 2018, pp. $90-93$.

[13] J. Shi, L. P. Jin, J. Li and Z. X. Fang, "A smart parking system based on NB-loT and third-party payment platform," in International Symposium on Communications and Information Technologies, 2017, pp. 1 - 5.

[14] S. A. R. Regi, "Remote monitoring of water level in industrial storage tanks using NB-loT," in ICCICT, Jan. 2018, pp. $1-4$.

[15] R. B. Srensen, D. M. Kim, J. J. Nielsen and P. Popovski, "Analysis of latency and MACLayer performance for class a LoRaWAN," IEEE Wireless Communications Letters, vol. 6, no. 5, pp. 566 - 569, Mar. 2018.

[16] M. Kuzlu,; M. Pipattanasomporn, R. Saifur, "Communication network requirements for major smart grid applications in HAN, NAN and WAN." Computer Networks 2014, 67, 74-88.

[17] Y. Azzougui , A. Recioui and A. Mansouri, "PMU Optimal Placement in Wide Area Monitoring Systems using Grey Wolf Optimization technique", Algerian Journal of Signals and Systems, Vol. 4, Issue 1, June 2019. pp:1-7.

[18] C37.118-2005, IEEE Standard for Synchrophasors for Power Systems, 2006.

[19] Y. Grainat, A. Recioui . "Application and Optimization of MIMO Communication in Wide Area Monitoring Systems," International Journal of Data Science ISSN 2722-2039, Vol. 1, No. 2, December 2020, pp. 82-98.

[20] Jianfeng, L.; Defu, J.; Xiaofei, Z. "DOA Estimation Based on Combined Unitary ESPRIT for Coprime MIMO Radar," IEEE Communications Letters 2017,vol. 21 n. 1, 9699.

[21] Recioui, A.; Bentarzi, H. "Application of a Galaxy-Based Search Algorithm to MIMO System Capacity Optimization," Arabian Journal for Science and Engineering 2016, vol. 41 n. 9, 3407-3414.

[22] A. RECIOUI et al., "Direction of Arrival Estimation for Interference Rejection in Mobile Communication Systems Employing Smart Antennas", 8th Mediterranean Microwave Symposium MMS'2008 at Damascus, Syria, 14 to 16 October 2008.

[23] A. RECIOUI and H. Bentarzi, "Optimization of Linear Array Antenna with Mutual Coupling consideration using Taguchi Method", The 11th international conference on sciences and techniques of automatic control and computer engineering -STA' 2010», Monastir, Tunisia December 19-21, 2010.

[24] Wu, Y.; Bergmans, J. W. M.; Attallah, S. "Effects of antenna correlation and mutual coupling on the carrier frequency offset estimation in MIMO systems." In Int. Conf. Wireless Commun. Netw. Mobile Computing (WiCOM), Chengdu, China, 23-25 Sept. 2010.

[25] A. Recioui, "Use of Spiral Optimization Technique to Enhance the Capacity of MIMO Communication Systems Employing One and Two-Dimensional Array Antennas", International conference on applied analysis and mathematical modelling, Yildiz Technical university, Istanbul, Turkey, June 8-12, 2015.

[26] A. Recioui, H. Bentarzi, Capacity Optimization of MIMO Wireless Communication Systems Using a Hybrid Genetic-Taguchi Algorithm, Wireless Personal Communications, Volume 71, Issue 2, 2013, pp 1003-1019

[27] A. Recioui, "Capacity Optimization of MIMO Systems Involving Conformal Antenna Arrays using a Search Group Algorithm", Algerian Journal of Signals and Systems, Vol. 5, Issue 4, December 2020. pp: 209-214.

[28] A. Recioui et al., "Combating Multiple Access Interference in Wireless Communication Systems Employing Smart Antennas", International conference on signals, systems and devices (SSD'09) in Djerba, Tunisia, 23 to 26 March 2009.

[29] H. Wei., D. Wang., H. Zhu., "Mutual coupling calibration for multiuser massive MIMO systems." IEEE Trans.Wireless Commun. 2016, vol. 15 n. 1, 606-619.

[30] I. S. Moskowitz, P. Cotae, M. H. Kang and P. N. Safier, "Capacity Approximations for a Deterministic MIMO Channel,"International Journal on Advances in Electrical and Computer Engineering, vol. 11, no. 3, (2011), pp. 3-10.

[31] ECE 476/ECE 501C/CS 513 - Wireless Communication Systems Winter 200 Lecture 1: Introduction

[32] Intel, Broadband Wireless: The New Era in Communications, Technical White Paper, 2004.

[33] T. S. Rappaport, A. Annamalai, R. M. Buehrer, and William H.Tranter, "Wireless communications: Past events and a future perspective,"IEEE Communications Magazine, vol. 40 n. 5:148\{161, May 2002.

[34] K. Su, "Efficient Maximum Likelihood detection for communication over Multiple Input Multiple Output channels ,"Laboratory for Communication Engineering ,Cambridge University Engineering Department,University of Cambridge, February 2005.

[35] X. H. Zhang; M. M. Zhang; F. F. Meng;; Y. Qiao; S. J. Xu; H. Senghout. "A Low-Power Wide-Area Network Information Monitoring System by Combining NB-IoT and LoRa". IEEE Internet Things J. 2019, 6, 590-598.

[36] S. Liu, C. Xia, and Z. Zhao, "A low-power realtime air quality monitoring system using Ipwan based on lora," in Solid-State and Integrated Circuit Technology (ICSICT), $201613^{\text {th }}$ IEEE International Conference on. IEEE, 2016, pp. 379-381.

[37] R. Sanchez-Iborra and M. D. Cano, "State of the art in LP-WAN solutions for industrial IoT services," Sensors (Switzerland), vol. 16, no. 5, 2016.

[38] B. Reynders, W. Meert, and S. Pollin, "Power and Spreading Factor Control in Low Power Wide Area Networks.", Belgium, May 2017.

[39] A. Waret, M. Kaneko, A. Guitton, and N. El Rachkidy, "LoRa Throughput Analysis with Imperfect Spreading Factor Orthogonality.", October , $3^{\text {rd }} 2018$

[40] B. Al Homssi, K. Dakic, S. Maselli, H. Wolf, S. Kandeepan, and A. Al-Hourani, "loT Network Design using Open-Source LoRa Coverage Emulator ". IEEE Access. 2021. 